\title{
A Vapor Based Microfluidic Flow Regulator
}

\author{
Wei Xu ${ }^{a}, 1$, Liang L. Wu ${ }^{*}, 1$, Yang Zhang, Hong Xue ${ }^{b}$, Guann-Pyng Li, and Mark Bachman \\ Department of Electrical Engineering and Computer Science, University of California, Irvine, CA \\ 92697, USA \\ aAlso with Department of Chemistry, University of North Carolina, Chapel Hill, NC 27599 \\ ${ }^{b}$ Department of Mechanical Engineering, California State Polytechnic University, Pomona, CA \\ 91768, USA
}

\begin{abstract}
We introduce a flow regulating technology that uses trapped air bubbles in a hydrophobic microfluidic channel. We present basic designs for flow regulators and flow valves using trapped air. Experiments have successfully demonstrated the capability of this technique for delivering constant and varying flow rate, and for on-off valving. This approach to valving provides a simple, yet effective way to monolithically integrate flow and valve control on polymer Lab-on-Chip devices.
\end{abstract}

\section{Keywords \\ Microflow regulator; Microfluidics; Microvalve; hydrophobic; Polymer PDMS; Lab-on-Chip; Concentration gradient}

\section{INTRODUCTION}

Miniaturized fluidic devices are of interest for many applications, including portable point of care fluid testing, high throughput chemistry, and micro-analytical instruments $1,2,3,4,5,6$. A critical requirement in microfluidic devices is the need to precisely meter fluid or control flow of fluid, something that is typically difficult to do at the microscale. Flow control devices including microflow regulators and microvalves have been developed based on a variety of actuation strategies such as electrostatically actuated MEMS structures ${ }^{7}$, pneumatic deformation of silicon and PDMS membranes ${ }^{8-9}$, piezoelectric flaps ${ }^{10}$, chemically and thermally responsive hydrogels ${ }^{11-} 12$, magnetic ferrofluids 13 and magneto-hydrodynamic fluid control14. Despite these many approaches, microfluidic flow control remains difficult to integrate easily on a microfluidic chip. Bubble-based microfluidic control devices have been demonstrated ${ }^{15}$, and may be useful for microfluidic applications since they are easy to fabricate and integrate on a small device, conform to irregular shaped walls (thus making good seals), and have no moving mechanical parts. The earliest successful application of using bubble for fluid control was in the bubble-jet printer which used rapidly heated bubbles as pumps ${ }^{16}$. Since then, several bubble based microvalves and pumps have been reported in the literature. For

(C) 2009 Elsevier B.V. All rights reserved.

*Corresponding author Tel: 1-949-824-5096, Fax: 1-949-824-3732, 1wu@uci.edu.

${ }^{1}$ Wei Xu and Liang Wu contributed equally to this work

Publisher's Disclaimer: This is a PDF file of an unedited manuscript that has been accepted for publication. As a service to our customers we are providing this early version of the manuscript. The manuscript will undergo copyediting, typesetting, and review of the resulting proof before it is published in its final citable form. Please note that during the production process errors may be discovered which could affect the content, and all legal disclaimers that apply to the journal pertain. 
example, Maxwell17 utilized thermal bubble nucleation method to develop an actuator with the application of bioparticle control. Hua15 created a bubble based microvalve by electrochemically generating bubbles inside the silicon microchannel to stop fluid flow. Wijngaart18 designed a novel thermal bubble based microvalve in which gas bubble was pretrapped in a large side chamber with a coil heater enabling bubble expansion to eventually block the channel. $\mathrm{Ki}^{19}$ utilized pressure barrier of bubble in the channel restriction region to form a passive bubble microvalve.

We report a bubble-based valve system that is simple in design and fabrication, can be individually addressed electronically, can be easily integrated into existing microfluidic devices without additional manufacturing steps, and requires very little footprint. In the current design, the bubble size, and thus the valve opening, is controlled by a temperature gradient in the microfluidic channel. A small hydrophobic cavity in a microfluidic channel is used to create a local trap for air, which subsequently becomes a bubble valve after filling the microfluidic device with fluid. By increasing the temperature of the fluid in the channel with respect to the body of the chip, we can move the bubble from the microcavity into the main channel region, and thus control the flow of fluid. Eventually, the bubble movement is sufficient to completely block the channel. We have found that this simple valve provides an easy, but surprisingly precise way to control the flow of fluid through a channel. We have demonstrated a flow control system using these valves.

\section{DESIGN}

To manipulate fluidic movements in a microchannel, many approaches have been exploited such as utilizing or altering the surface properties of microchannels ${ }^{20-21}$, varying channel designs $^{22}$, using nanoparticles for optofluidic control ${ }^{23}$, etc. In our device, we utilized a well known phenomenon of hydrophobic microchannels ${ }^{24}$ in the geometric design to create an air pocket that is used to control the fluid flow. A schematic of the vapor based microflow regulator is shown in Fig. 1. We fabricated a polymer microfluidic channel, $150 \mu \mathrm{m} \times 50 \mu \mathrm{m}$, in poly (dimethylsiloxane) (PDMS) with the valve geometry. The valve consisted of a pair of identical microconcave structures, which we called air cavities $(l \times w=150 \mu \mathrm{m} \times 80 \mu \mathrm{m})$, attached to a narrow channel $(r=50 \mu \mathrm{m})$. Following the valve region, the microfluidic channel returned to its original width $(d=150 \mu \mathrm{m})$. An external heater, gold meander traces $(0.25 \mu \mathrm{m}$ thick, 50 $\mu \mathrm{m}$ wide) lithographically patterned onto a glass slide, was then placed at the upstream of the channel to generate a small temperature gradient between the incoming fluid and the valve regions.

The operation of the device is illustrated in Fig. 2. In the first step (Fig. 2a), water (red) was injected into the microchannel with the external heater turned off. While filling the main section of the channel, surface tension of water prevented the fluid from entering the microconcave structures due to the hydrophobic nature of the $\mathrm{PDMS}^{24}$, leaving trapped air inside the cavities of the valve. The surface tension boundary (we call "virtual walls") between air bubbles and water supported the fluid flow and was stable over several weeks, as long as the channels stayed hydrated. During operation, the bubbles moved from the air cavities into the main channel region through a process of mass transfer $25^{-} 26$. To initiate the transfer, the external heater was turned on which raised the temperature of the water in the channels by a few degree Celsius, as shown in Fig 2e. The temperature was estimated by infrared (IR) thermal mapping using an IR Camera (service provided by MEFAS, Inc. Irvine, CA) with a thermal resolution of $0.5^{\circ} \mathrm{C}$ and a spatial resolution of $5 \mu \mathrm{m}$. Warm vapor from the heated fluid then evaporated from the liquid-vapor interface into the air filled cavities, which remained near room temperature. Fig. $2 \mathrm{~b}$ shows the initiation of condensation when warm vapor saturated the small cavity regions. This condensation caused the trapped air to be displaced into the main channel, thus restricting the flow channel cross section and increasing flow resistance, as shown in Fig. 2c. When this 
process of mass transfer was allowed to proceed long enough, the air bubbles coalesced and formed a continuous physical air barrier, which completely stopped the water flow as shown in Fig. 2d.

The flow regulating and valving of the device primarily depends on the mass transfer mediated by the temperature gradient. Air expansion from the ideal gas law and PDMS polymer outgassing can partially contribute to the growth of bubbles and they were experimentally tested, as described in Sec. IV. The valve can be modeled by an air-filled cavity with an opening to water. When the entire system is at constant temperature, the vapor/liquid evaporation/ condensation from the water and wall interfaces is at equilibrium and there is no net change in the bubble. Once the water is heated, a temperature gradient forms so that the liquid has a higher temperature than the wall. The net effect is that vapor generated from high temperature water boundary condensates at the cooler wall boundary forming water droplets, which in turn displace the air bubble. The dependence of mass flux $(\Gamma)$ on local temperature is given by:

$$
\Gamma=C \sqrt{\frac{M}{2 \pi R T}} \exp \left(-\frac{\Delta h}{R T}\right),
$$

where $\mathrm{M}$ stands for the molecular weight of water molecule, $\mathrm{R}$ the gas constant, $\Delta \mathrm{h}$ the latent heat of water vaporization $\left(44 \mathrm{~kJ} / \mathrm{mol}\right.$ at $25^{\circ} \mathrm{C}$ ) and $\mathrm{C}$ is a systematic constant ${ }^{27}, 28,29$. The net transfer rate is the difference between flux at the walls and at the water boundary, which depends on the difference in temperature.

The design of an abrupt reduction of channel width following the air cavities structures prevented the flushing of displaced air bubbles down stream during the flow regulating process. We observed a corresponding reduction in flow rates when the external heater was turned on, indicating a smooth and continuous evaporation and condensation process. On the other hand, after the heater was turned off, the direction of the temperature gradient was reversed when fresh solution from the reservoir flowed through the channel at a lower temperature than the previously heated air in the cavities. Therefore, many of the condensed drops were reevaporated into main channel resulting in a return of the air bubbles back into the cavities and restoring the channel width. During this procedure, flow resistance was reduced and the flow rate increased while the corresponding vapor pressure was reduced. Therefore, by turning on and off the external heater, the fluid flow can be regulated by controlling the location of the air boundaries.

This vapor based microflow regulator can also be used as a single-shot, irreversible microfluidic stop valve if one desires by continuing the bubble movement until the two air bubbles coalesce. However, if enough pressure is applied in the channel, typically $40 \mathrm{~cm}$ $\mathrm{H}_{2} \mathrm{O}$ for our devices, the bubbles can be flushed away and the device can be re-used.

To demonstrate the bubble valves, we designed a simple microfluidic "T" channel device, as shown in Fig. 3. One channel of the device had a bubble flow regulator while the second did not. The two channels joined to a single channel, and the relative flow rates of the two channels were readily monitored by observing the location of the Laminar flow boundary between the two fluids in the center channel.

\section{EXPEIMENTAL METHODS}

The fabrication of the device was a single mask PDMS replica process. A micromachined silicon mold was prepared by standard microlithography and followed by deep reactive ion etch. After fabrication, the mold was soaked in Sigma-cote (Sigma Chemical Co., St. Louis, 
MO) overnight to ease the release of PDMS after casting. PDMS mixture (Dow Corning, Midland, MI) was prepared by mixing polymer base and curing agent at a weight ratio of 10:1. The degassed PDMS mixture was poured onto the mold, cured in oven at $85^{\circ} \mathrm{C}$ for 15 minutes and followed by mold release. Meanwhile, the external heater was fabricated by patterning gold meander traces of $250 \mathrm{~nm}$ in thickness and $50 \mu \mathrm{m}$ in width onto a flat microscope glass slide using traditional photolithography. A thin layer (approximately $10 \mu \mathrm{m}$ ) of PDMS was then spin-coated and cured onto the glass slide covering the gold heater. The two PDMS surfaces were irreversibly bonded by treating with oxygen plasma for 10 seconds (120 mTorr of pressure, $75 \mathrm{~mW}$ of power). The chip was left in air overnight to reverse the temporary hydrophilicity of the PDMS surface induced by oxygen plasma ${ }^{30}$.

To test the performance of the vapor based microflow regulator, a microfluidic device was constructed as shown in Fig. 3. It consisted of two parallel channels $(150 \mu \mathrm{m}$ in width and 50 $\mu \mathrm{m}$ in depth) with one of them as a reference channel containing no valve structures. The other channel consisted of a paired air cavity structures (as described above) filled with air. The two channels were joined by a " $T$ " connection allowing them to share a common fluidic waste channel. For demonstration purpose, a red food dye solution (Escofoods.com, 20\% by volume) was used as a visible indicator. Water was injected through both channels, red solution in the flow regulating channel (A) and clear water in the reference channel (B), as shown in Fig 3. Constant fluid pressure was maintained by providing a height difference between inlet reservoirs and the outlet waste reservoir. The height of the inlet reservoirs with respect to the waste reservoir was 29 centimeters for the regulating side (A) and 20 centimeters for the reference side (B). The total flow rate was measured to be $4.8 \mu \mathrm{L} / \mathrm{min}$. The gold meander heater was approximately $0.6 \mathrm{~mm}$ upstream of the valve structure connected to a power supply. Videos of fluid flow were recorded by a NIKON Coolpix 990 camera mounted on an inverted microscope (Olympus, BH2-UMA).

At the beginning of the experiment, the pressure difference between reservoirs A and B was adjusted so that clear water flow was just visible below the $\mathrm{T}$ junction and red dye flow occupied $100 \%$ of the region, Fig. 3. This initial state applied to all our microflow regulator experiments. Trapped air bubbles remained in their cavities for many hours with pressure drops ranging from a few centimeters to over $30 \mathrm{~cm}$ of water. However, these bubbles would eventually shrink after several hours to several days depending on the valve design (more pairs of air cavities would sustain the valve longer), which was due to the porous nature of PDMS that allowed gasses to diffuse into the material. Relative flow rates of the water streams were determined by monitoring the position of the laminar flow boundary between the regulated flow (red) and the reference flow (clear) below the $\mathrm{T}$ junction. The position of laminar flow boundary was verified by analyzing the image pixels intensity change. By integrating over the parabolic flow cross sectional profile, we determined relative flow rate change during microflow regulator operation.

\section{VALVING MECHANISM STUDIES}

To test our observation that a temperature gradient is required for valve operation, we subjected the microfluidic devices to a uniform change in temperature. We placed the device inside a transparent incubator and observed the bubble valves as the temperature in the incubator was increased. The observations are shown in Fig. 4. As the incubator temperature increased to 32 ${ }^{\circ} \mathrm{C}$, the air bubble started to expand as shown in Fig. 4a. However, unlike the case when a temperature gradient was applied (Fig. 4b), the uniformly heated device did not show water condensation in the cavities. When the incubator was turned off and the device allowed to cool, the air in the cavities did not shrink, but rather continued to expand. 
We can postulate three hypotheses for the expansion or movement of the air bubble from the cavity into the flow channel: 1) the bubble expands due to ideal gas law heating, 2) the bubble expands due to PDMS outgassing, and 3) the bubble moves due to evaporation and recondensation of water in the air cavity (for temperature gradient). According to the ideal gas law, the change in volume is directly proportional to temperature. In our uniform heating experiment, the temperature was raised $13 \mathrm{~K}$, so the expected increase in volume is $4.4 \%$. However, we observed the volume expansion of the bubbles to be approximately $30 \%$ in the device indicating that the ideal gas law was only a small factor contributing to the air movement in this experiment.

When temperature is increased, PDMS is known to outgas due to decreased solubility of gas $^{31}$. To study the outgassing effect, we filled a PDMS microchannel $(150 \mu \mathrm{m} \times 50 \mu \mathrm{m}$ cross section) with water and a trapped air bubble. The chip was placed in the incubator with one end of the channel sealed, and the other opened to air to allow displacement of the water. We observed the bubble growth as we increased temperature; the bubble size as a function of temperature is shown in Fig. 4c. The data indicated a hysteresis effect; the air bubble continued expansion after the incubator was switched off as shown in Fig. 4c, and never returned to its original size. The two arrows indicated the direction of the temperature change and the air bubble had grown five times its original volume size. Clearly, PDMS outgassing is a major mechanism for bubble growth under uniform heating conditions.

To validate the concept that gradient mediated mass transfer can be a driving mechanism to actuate the air bubble in our valves, we performed an additional experiment by filling a glass micro-capillary with water while trapping a known size air bubble inside. We sealed one end of the glass capillary and left the other open to air to allow free movement of the water as the bubble expanded or moved. We used the glass capillary to eliminate outgassing, so that the mechanism of mass transfer could be studied. We placed a Peltier heater underneath the sealed end of the capillary near the air bubble, and a metal heat sink the other opened end. The temperature of the Peltier heater and the heat sink were monitored simultaneously. We observed the formation of condensation drops after the heater was turned on; we have plotted the net displacement of the bubble/water boundary as a function of temperature in Fig. 4d. When the heater was turned off, the air bubble contracted but was not restored completely to its original size because some condensed water droplets remained in the air cavity. This experiment clearly shows that a small thermal gradient can produce evaporation and condensation in a small air cavity, resulting in movement of the air bubble.

\section{FLOW CHARACTERIZATION}

A series of experiments were conducted including flow response characterizations, flow rate stability testing with feedback control, and a single-shot valve display to demonstrate the capability of such device regulating flow at small scale and ease of operation. Furthermore, we used the valves to generate a uniform concentration gradient to demonstrate the potential of this flow regulator for biochemical assays.

The flow regulator response was estimated by pulsing the heater and observing the flow stream in the common channel below the " $T$ " junction, as described in the experimental section. At $\mathrm{t}<0$, the heater was turned off, the pressure difference of two inlet reservoirs A and B was adjusted so that most of the flow in the "T" channel came primarily from the A reservoir (passing through the valve). At $\mathrm{t}>0$, then the heater was turned on at $0.78 \mathrm{~V}$ and $25 \mathrm{~mA}$. The average resistance of the external gold heater was $32 \Omega$. Since many factors affect the heating of the fluid, including the location and shape of the external heater, its thermal contact with the PDMS, the thermal isolation of the chip, and the flow rate of the fluid, we cannot use first principles to correlate heater power with corresponding temperature increase in the flow. 
However, through infrared (IR) thermal mapping, we estimate that the fluid temperature next to the valve was increased approximately up to $8.3^{\circ} \mathrm{C}$ for the complete valving scenario.

Droplet condensation in the air cavities was immediately observed after heat was applied, and bubbles began to expand to the main section of the channel, reducing the effective channel width and flow rate (Fig. 5a). The Laminar flow boundary in the main channel shifted from the reference channel side toward the microflow regulator channel side in direct response to the applied current as seen in Fig. 5b. The flow state was then returned to its original position by turning off the heater after the boundary shifted to a pre-marked line on the screen (dotted line on Fig. 5b). With the heater turned off, we immediately observed a partial reversal of the mass transfer phenomenon--air bubbles were restored into cavities as water drops re-condensed into the main flow. During this period, the water flow, which came from the external reservoirs, was temporarily at a lower temperature than the air cavities. We repeated this flow response process several times and have plotted the red dye flow rate vs. time in the main channel (Fig. 6a). The red dye flow rate was determined from the observed Laminar flow boundary and total flow rate by assuming a parabolic flow profile over the cross section of the channel. The initial response time to actuate the valve was typically $15-40$ seconds depending on the location of the air/liquid boundary. During the experiment, the response time to actuate or halt the movement of laminar flow boundary in accordance to heat input was within a few seconds.

Fig. $6 \mathrm{~b}$ shows that the flow rate was controllable when a feedback control was applied. To investigate the regulator's ability of delivering the flow rate at preset points, a second flow rate measurement was performed with feedback control conducted. The flow boundary was easily kept constant at a pre-marked line on the screen by repetitively switching the external heater on or off as needed, and the response time for actuating the laminar flow boundary was within a second. The device was more sensitive in response as the separation of the two valving bubbles was reduced. The result is shown in Fig. $6 \mathrm{~b}$ with a constant flow rate at $2.4 \mu \mathrm{L} / \mathrm{min}$ and minimal fluctuation caused by the inaccuracy of manual control mode (this can be reduced by utilizing an automatic feedback control circuit). Thus, this novel vapor based microflow regulator is capable of providing flow rate control as indicated by Fig. $6 \mathrm{~b}$.

Another experiment was performed to demonstrate the flow regulator as a one-shot valve to completely stop the flow in a microfluidic channel. This was realized by leaving the heater on until the two bubbles coalesced, Fig. 7a, after which the heater was turned off. Once the coalescence happened, a pressure barrier was established and flow was stopped. A red dye flow rate response curve was plotted with time at $1.46 \mathrm{~V}$ and $45 \mathrm{~mA}$ as shown in Fig. $7 \mathrm{~b}$. The greater the input power added to the heater, the faster the regulator responded, as seen in Fig. 7. Pressure experiments showed that the barrier could withstand a maximum of $40 \mathrm{~cm}$ water pressure before the bubble pushed downstream and the flow resumed.

Finally, an application of the regulator was demonstrated by designing a microfluidic concentration gradient chip. The generation of concentration gradients is very important in many chemical and biological applications such as protein crystallization ${ }^{32}$ and cell culturing ${ }^{33}$. Current approaches to generating concentration gradient on microfluidic chip utilize networks of channels to produce a preprogrammed gradient, or utilize external, computer controlled actuators. Using this simple vapor based microflow regulator, we designed a low cost, easily fabricated microfluidic concentration gradient chip, as seen in Fig. 8. The concentration gradient chip consisted of two branch channels converging at a T junction. After the $\mathrm{T}$ junction, the channel formed a serpentine pattern. To provide a direct visual view of the concentration gradient, the red dye solution was injected into the flow regulator channel while clear water flow was injected into the other. The pressure head of the red dyed water flow was maintained at $8 \mathrm{~cm}$ water pressure. The pressure difference of the red dyed water and clear water was adjusted as previously described. After filling the first half of the serpentine channel 
with only red dye solution, the relative flow rate was then gradually adjusted with appropriate heating provided, and two flows mixed by diffusion at the downstream of the T junction. A clear comparison is shown in Fig. 8 between two sections of the channel. A continuously varying concentration was set up within a matter of minutes in half of the serpentine regions which can be seen as different color magnitudes of the dye.

\section{CONCLUSIONS}

In this paper, we introduce a novel vapor based microflow regulator. By taking the advantage of hydrophobic surface properties of polymers such as PDMS, this microflow regulator successfully traps air bubbles which are then used as flow rate control elements. Actuation is accomplished by creating a temperature gradient in the microfluidic channel and the adjoining cavities of the valve, and bubbles move by condensation of vapor from the channels. Compared with other microfluidic valves, this regulator has advantages of simple design, easy fabrication and integration, easy control, and low cost. We demonstrated that this flow regulator can maintain constant flow rate through feedback control, and the flow regulation can be achieved in a programmable way as long as the proper control circuitry is applied. If the two bubbles are allowed to coalesce, the regulator becomes a closed valve and permanently switches off the flow. Thus the single device can provide both regulating and latched valving functions, which is helpful in reducing the size and complexity of the microfluidic device. We believe this microflow regulator has many potential applications for microfluidic systems. As an example application, we designed a microfluidic concentration gradient chip and demonstrated using this valving technique. The result shows the successful generation of a concentration gradient.

\section{Acknowledgments}

The authors would like to express special thanks to Dr. Yuli Wang for his valuable discussion. The authors also would like to thank Ruisheng Chang, Trang Bui, Ngoc-Thanh Pham and the staff of the UC Irvine Integrated Nanosystems Research Facility for their microfabrication assistance. This work was supported by the following grants: Defense Advanced Research Project Agency (contract HR0011-06-1-0050), National Institute of Health (contract 7 R01 EB004436-05), and IUCRP-UC Discovery (contract ELE06-10273).

\section{References}

1. Man, PF.; Mastrangelo, CH.; Burns, MA.; Burke, DT. Microfabriated capillary-driven stop valve and sample injector; IEEE MEMS Conference; Heidelberg, Germany. Jan. 25-29, (1998); p. 45-50.

2. Harrison DJ, Manz A, Fan Z, Ludi H, Widmer HM. Capillary electrophoresis and sample injection systems integrated on a planar glass chip. Anal. Chem 1992;64:1926-1932.

3. Figeys D, Pinto D. Laboralory-on-a-Chip: a revolution in biological and medical sciences. Anal. Chem 2000;72:A330-A335.

4. Chou H-P, et al. A microfabricated device for sizing and sorting DNA molecules. Proc. Nat. Acad. Sci 1999;96:11-13. [PubMed: 9874762]

5. Weigla BH, Bardella RL, Cabrerab CR. Lab-on-a-chip for drug development. Advanced Drug Delivery Reviews 2003;55:349-377. [PubMed: 12628321]

6. Arquint P, et al. Micromachined analyzers on a silicon chip. Clin. Chem 1994;40:1805-1809. [PubMed: 8070105]

7. Cabuz, E., et al. Mems-based Flow Controller for Flow Cytometry; Proc. 2002 Solid-State Sensors, Actuator and Microsystems Workshop; Hilton Head Island, SC. 2002. p. 110-111.

8. Cousseau, P., et al. Improved Micro-flow Regulator for Drug Delivery Systems; Proceedings of 14th IEEE International Conference on Micro Electro Mechanical Systems; Interlaken, Switzerland. 2001. p. 527-530. 
9. Yang, B.; Levis, JW.; Lin, Q. A PDMS-based constant-flowrate microfluidic control device; Proceedings of 17th IEEE International Conference on Micro Electro Mechanical Systems; Maastricht, Netherlands. 2004. p. 379-382.

10. Andersson H, van der Wijngaart W, Nilsson P, Enoksson P, Stemme G. A valve-less diffuser micropump for microfluidic analytical systems. Sensors and Actuators B, Chem 2001;72:259-265.

11. Lei M, Salim A, Siegel RA, Ziaie B. A hydrogel-actuated microvalve for smart flow control. IEMBS '04 26th Annual International Conference of the IEEE 2004;3:2041-2044.

12. Luo Q, Mutlu S, Gianchandani YB, Svec F, Frechet JM. Monolithic valves for microfluidic chips based on thermoresponsive polymer gels. Electrophoresis 2003;24:3694-3702. [PubMed: 14613195]

13. Hartshorne H, Backhouse CJ, Lee WE. Ferrofluid-based microchip pump and valve. Sensors and Actuators B, Chem 2004;99:592-600.

14. Bau HH, Zhu J, Qian S, Xiang Y. A magneto-hydrodynamically controlled fluidic network. Sensors and Actuators B, Chem 2003;88:205-216.

15. Hua SZ, Sachs F, Yang DX, Chopra HD. Microfluidic actuation using electrochemically generated bubbles. Anal. Chem 2002;74:6392-6396. [PubMed: 12510764]

16. Beatty, CC. A chronology of thermal ink-jet structures; Proc. Solid-State Sensor and Actuator Workshop; Hilton Head, SC. June 13-16 (1996); p. 200-204.

17. Maxwell RB, Gerhardt AL, Toner M, Gray ML, Schmidt MA. A microbubble-powered bioparticle actuator. J. of MEMS 2003;12:630-639.

18. van der Wijngaart, W.; Chugh, D.; Man, E.; Melin, J.; Stemme, G. A low temperature thermopneumatic gas bubble valve; $19^{\text {th }}$ International Conference on MEMS 2006; Istanbul, Turkey. Jan. 22-26. 2006 p. 198-201.

19. Ki, Y-SL.; Kharouf, M.; van Lintel, HTG.; Haller, M.; Renaud, P. Bubble engineering for biomedical valving applications; 1st Annual International Conference on Microtechnologies in Medicine and Biology; Lyon, France. Oct. 12-14. 2000 p. 390-393.

20. Zhao B, et al. Surface-Directed Liquid Flow Inside Microchannels. Science 2001;291:1023-1026. [PubMed: 11161212]

21. Delamarche E, et al. Microfluidics for processing surfaces and miniaturizing biological assays. Advanced Materials 2005;17:2911-2933.

22. Melin J, et al. Behaviour and design considerations for continuous flow closed-open-closed liquid microchannels. Lab Chip 2005;5:682-686. [PubMed: 15915262]

23. Liu GL, et al. Optofluidic control using photothermal nanoparticles. Nat. Mater 2005;5:27-32. [PubMed: 16362056]

24. Chen Y, Chen Z, Zhao Y, Nelson JS, Bachman M, Chiang Y-M, Chu C, Li G-P. Test channels for flow characterization of processed plastic microchannels. MRS Symposium Proceedings, Materials Science of Microelectromechanical Systems (MEMS) Devices II 2000;605:253-259.

25. Xu, W.; Xue, H.; Bachman, M.; Li, G-P. Mass transport phenomena in superhydrophobic surfaces; the 3rd Annual Integrated Nanosystems Design, Synthesis \& Applications Conference; Pasadena, CA. Sept. 22-24. 2004

26. Xu, W.; Xue, H.; Bachman, M.; Li, G-P. Virtual walls in microchannels; Proceedings of the $28^{\text {th }}$ IEEE EMBS Annual International Conference; New York City, NY. Aug. 30. Sept. 30. 2006 p. 2840-2843.

27. Langmuir I. the vapor pressure of metallic tungsten. the Physical Review 1913;II:329-342.

28. Fukuta N, Walter LA. Kinetics of hydrometeor growth from a vapor-spherical model. J. Atmos. Sci 1970;27:1160-1172.

29. Pruppacher, HR.; Klett, JD. Microphysics of Clouds and Precipitation. Kluwer Academic Publishers; 1997. Chapter 12

30. McDonald JC, et al. Fabrication of microfluidic systems in poly(dimethylsiloxane). Electrophoresis 2000;21:27-40. [PubMed: 10634468]

31. Boscaini E, Alexander ML, Prazeller P, Märk TD. Investigation of fundamental physical properties of a polydimethylsiloxane (PDMS) membrane using a proton transfer reaction mass spectrometer (PTRMS). Int. J. of Mass Spec 2004;239:179-186. 
32. Zheng B, Roach LS, Ismagilov RF. Screening of protein crystallization conditions on a microfluidic chip using nanoliter-size droplets. J. Am. Chem. Soc 2003;125:11170-11171. [PubMed: 16220918]

33. Chung BG, et al. Human neural stem cell grown and differentiation in a gradient-generating microfluidic device. Lab Chip 2005;5:401-406. [PubMed: 15791337] 


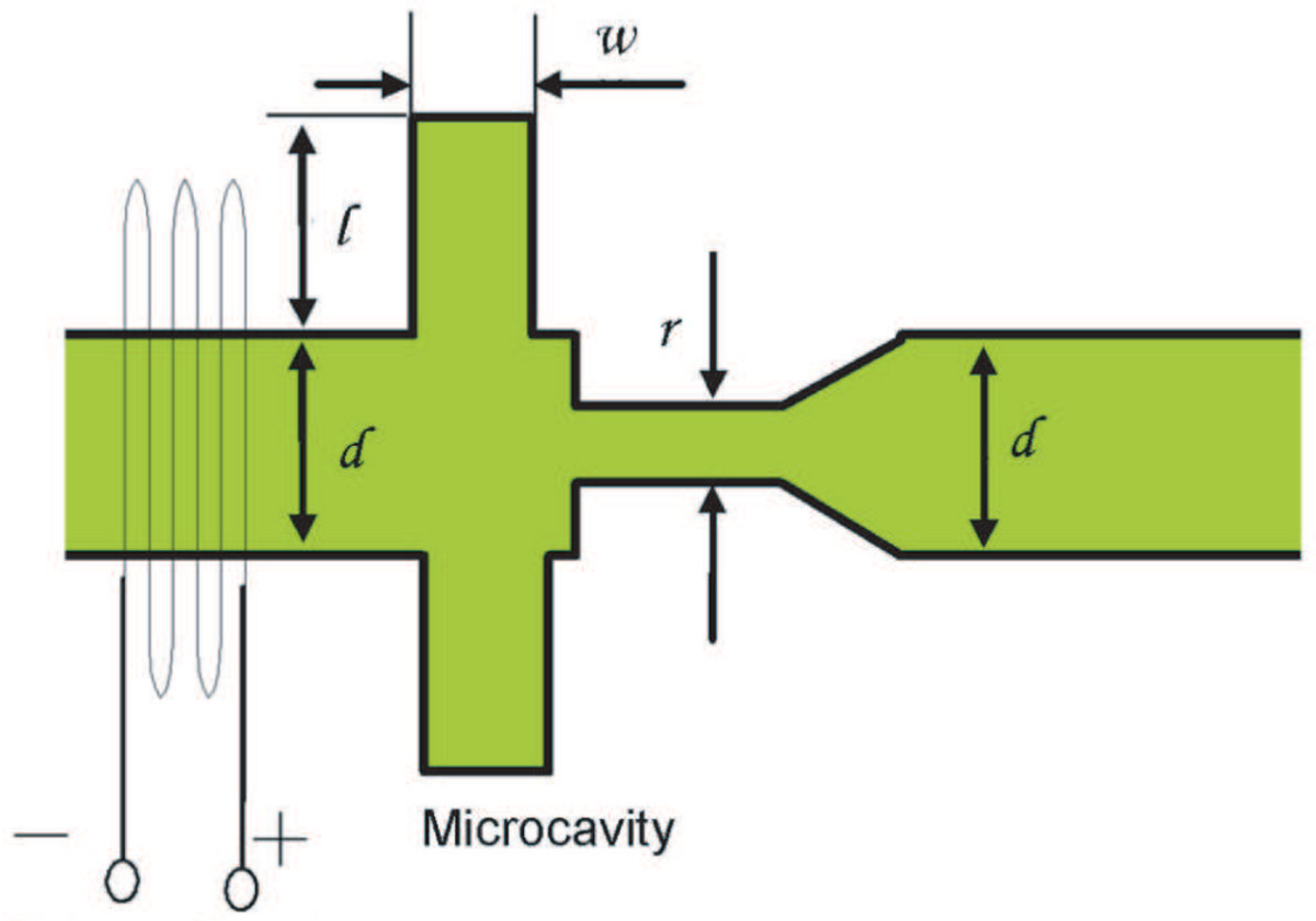

\section{External heater}

Fig. 1.

Schematic of overall vapor based microvalve test device. 

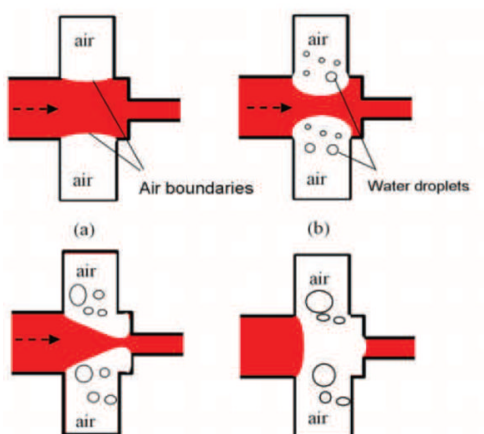

(c)

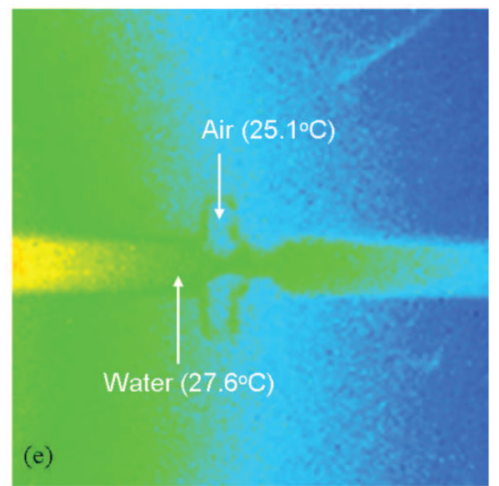

Fig. 2.

Schematic view of the operation of the vapor based microflow regulator. (a) Fluid (shown as red) is filled in the channel while external heater is turned off. (b) After the heater is turned on, water droplets condense in the cavity structures and air boundaries are pushed into the main section of the channel. (c) Abrupt reduction of the channel width prevents air bubbles from being flushed by water flow. (d) Air bubbles coalesce, completely blocking the channel, and water flow is stopped. Some of the condensed droplets have fused into larger ones. (e) An infrared thermal image of the device exhibiting the temperature difference between air-filled cavities and water in the microchannel. The device temperature is at $22.9^{\circ} \mathrm{C}$ before applying heat. Both water and air temperature have elevated to $27.6^{\circ} \mathrm{C}$ and $25.1^{\circ} \mathrm{C}$ respectively resulting in a $2.5^{\circ} \mathrm{C}$ temperature difference, which induces the evaporation and condensation of water into the cavities. 


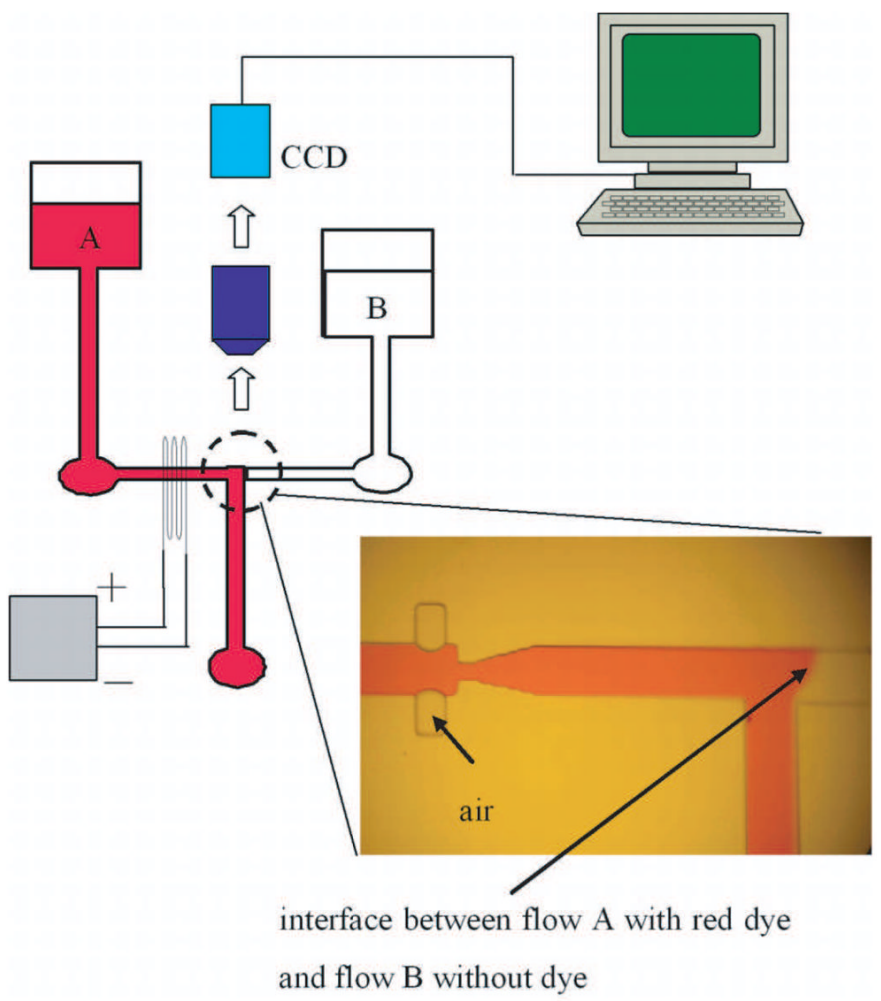

Fig. 3.

Diagram of the experimental set-up for microvalve regulator operation. Relative flow rate is determined based on the location of the laminar flow boundary between the two fluids. 

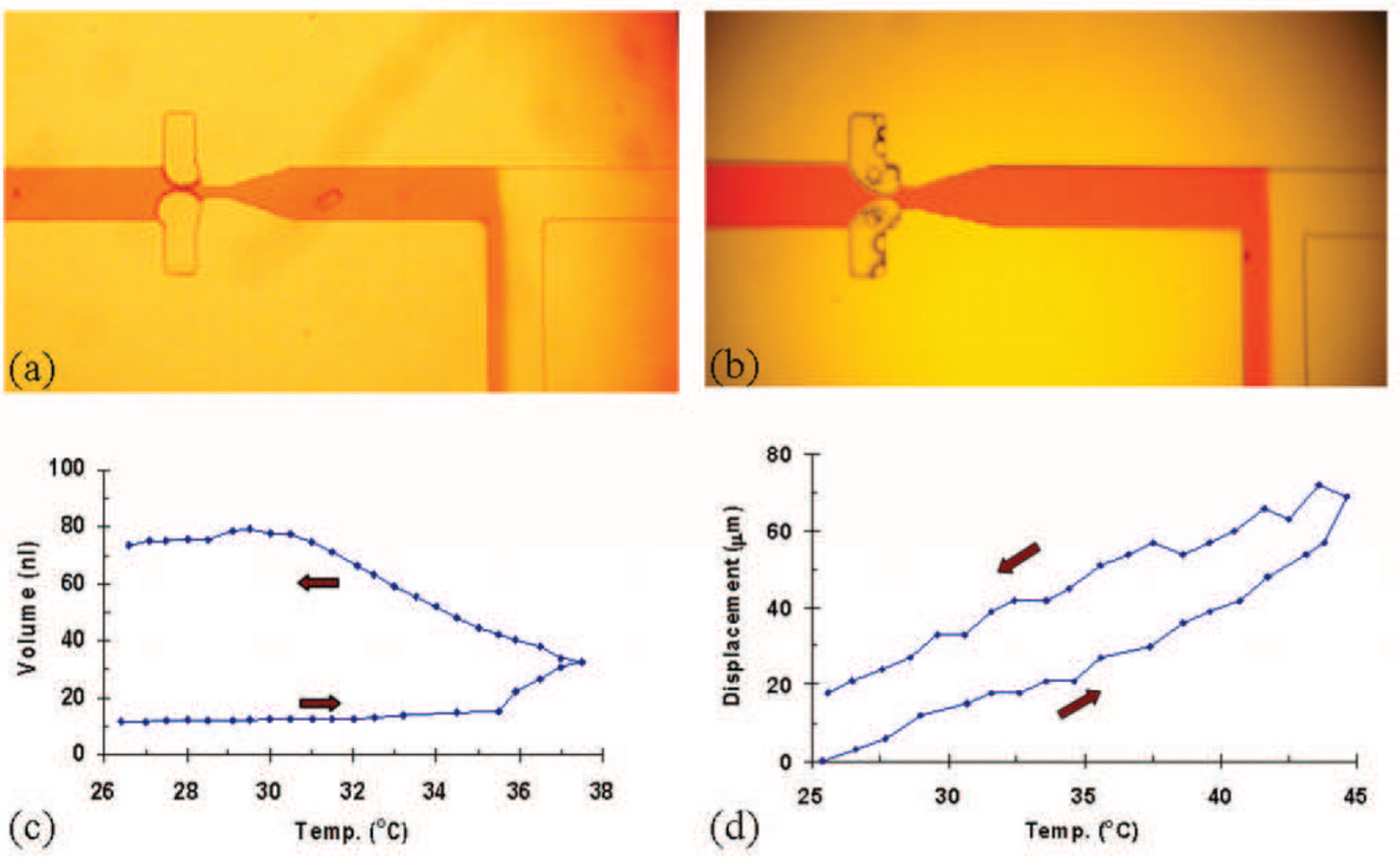

Fig. 4.

Comparison of two valving scenarios under (a) uniform heating and (b) temperature gradient mediated mass transfer. (c) Study of air expansion in PDMS with uniform heat applied. Two red arrows indicate the direction of temperature change. PDMS outgassing is evident from the hysteresis curve where the air volume increased to five times its original size after applying heat. (d) Study of air movement in a glass capillary tube under gradient mediated mass transfer. Air bubble was displaced by water condensate during heating; the volume was not completely restored during cooling, due to water that remained in the air region. 

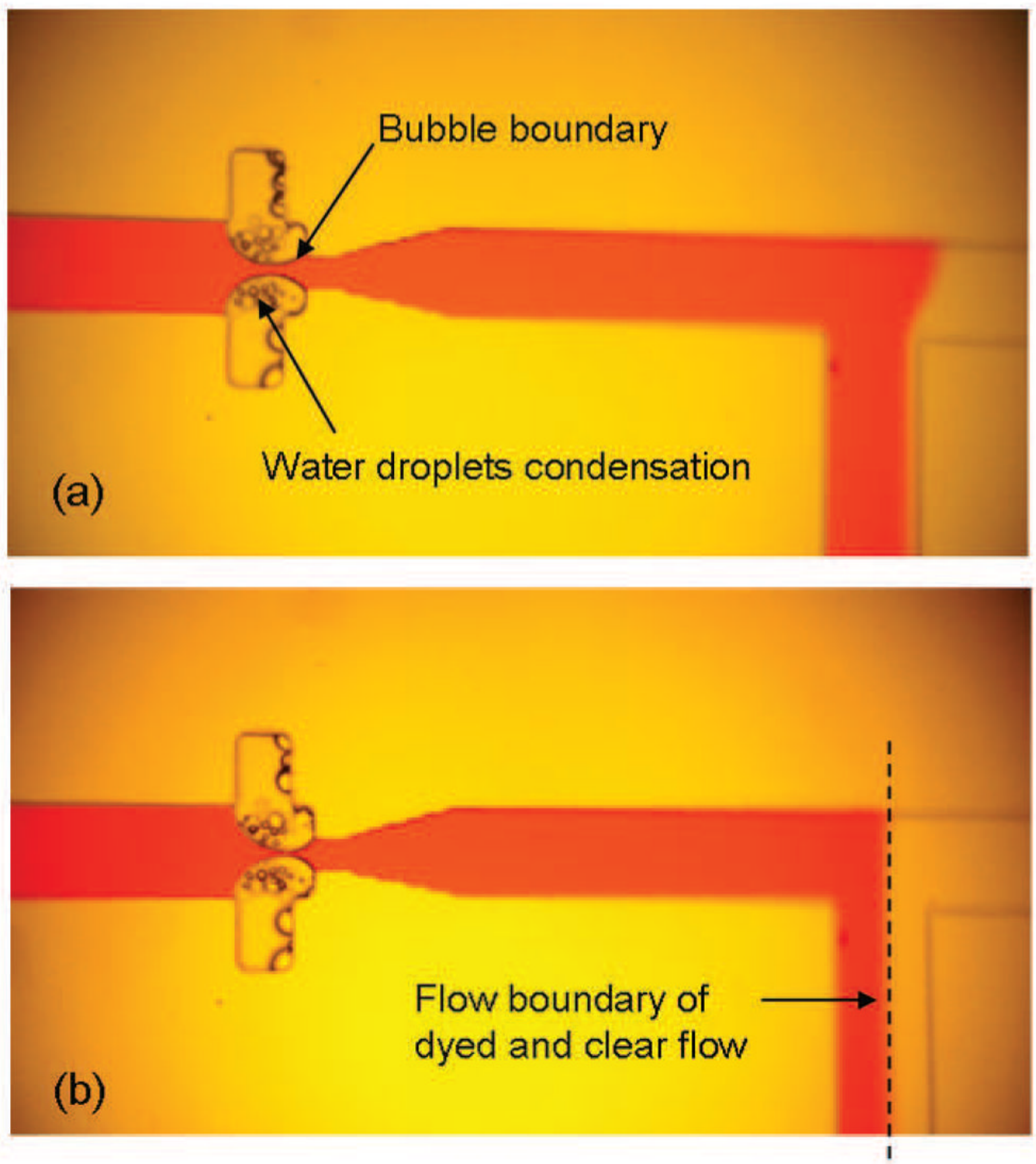

Fig. 5.

Images of vapor based microvalve regulator. First photograph (a) shows initial condensation of droplets. Second photograph (b) shows reduction in flow rate after bubbles have moved sufficiently far into the main section of the channel. The condensation drops are transparent (not dyed), since they are distilled from the solution. 


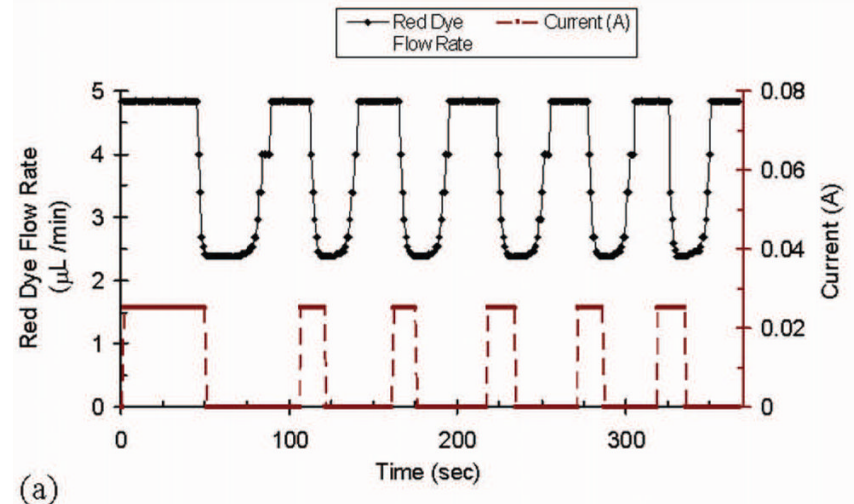

(a)

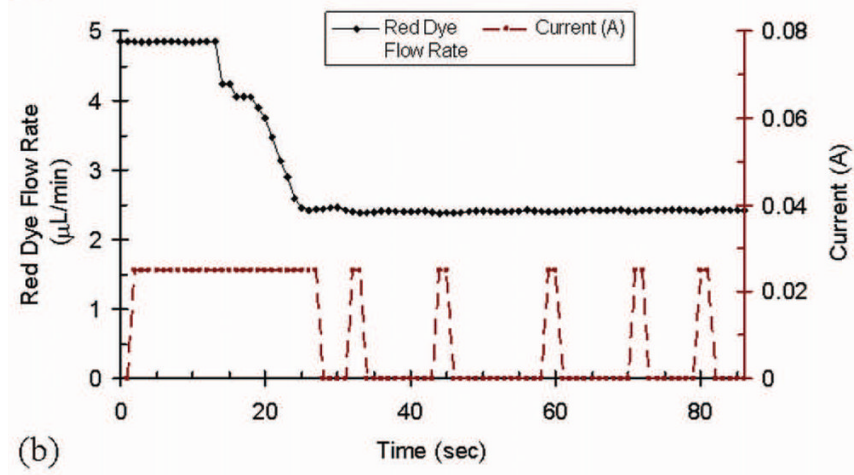

Fig. 6.

(a) Response of the flow regulator represented by change of red dye flow rate with time below the $\mathrm{T}$ junction region. The heater is turned on at $\mathrm{t}=0$ and the input current of external heater is $25 \mathrm{~mA}$. The heater is turned off when the laminar flow boundary reaches the pre-marked line and on again when red dye restores to the initial flow rate. The response time to actuate or halt the movement of laminar flow boundary in accordance to heat input is within a few seconds (b) Flow rate controlled by vapor based microvalve with manual feedback control to maintain a constant relative flow rate in the channel at $2.4 \mu \mathrm{l} / \mathrm{min}$. 


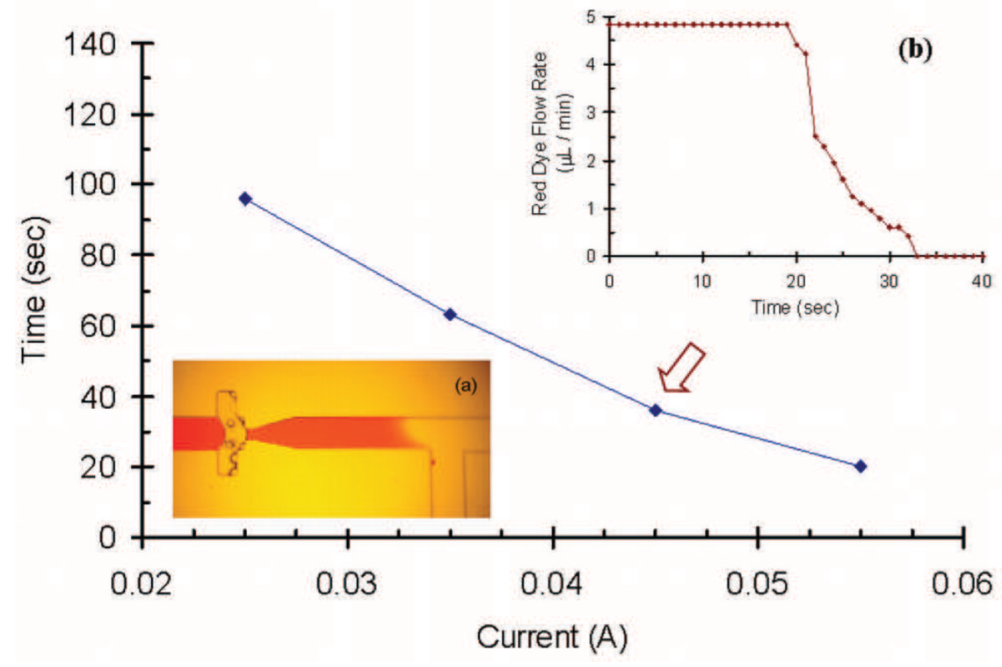

Fig.7.

One-shot valve operation demonstrating the actuation speed for the valve varying with input current. The valve is easily shut off in less than 20 seconds at $55 \mathrm{~mA}$. Valve can be re-opened after $40 \mathrm{~cm}$ water pressure is applied. (a) Continuous application of heat results in a permanent "off" state for the vapor based microvalve. (b) A typical red dye flow rate change vs. time in the $\mathrm{T}$ junction area at $45 \mathrm{~mA}$. 


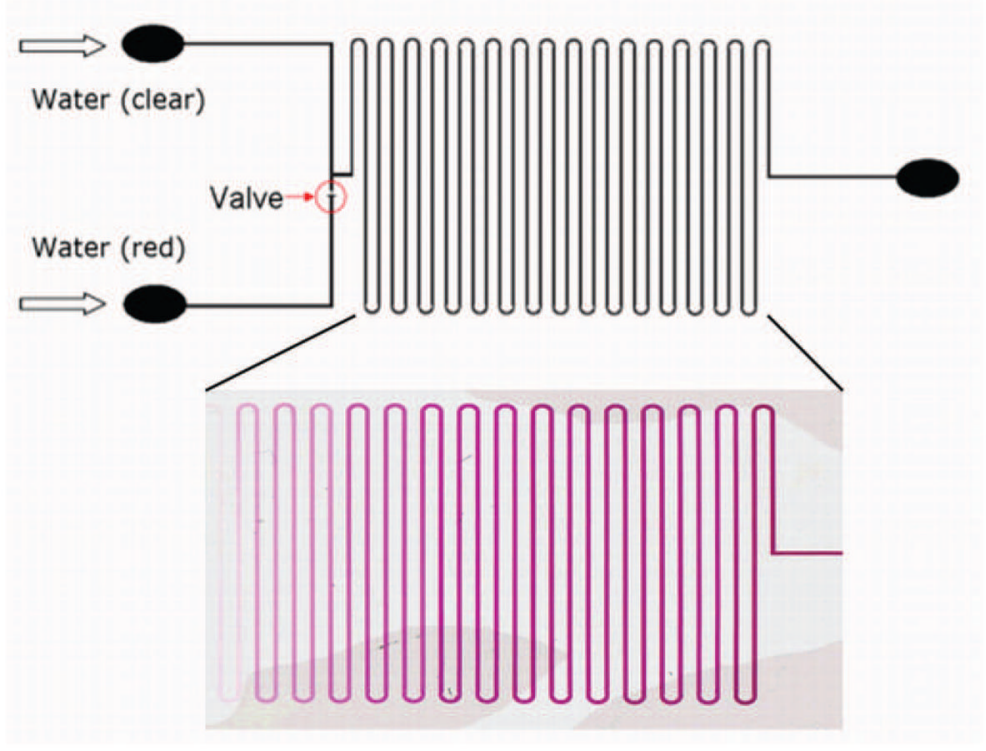

Fig. 8.

Schematic of microfluidic concentration gradient chip utilizing vapor based microflow regulation (top). A macroscopic picture of the PDMS device with serpentine channel (bottom) shows the result of concentration gradient generation caused by continually changing the bubble valve as water flowing through the device. After flow is stopped, the fluid in the channel has a concentration that varies along the length of the channel. 